\title{
Stoichiometry and intracellular fate of TRIM-containing TCR complexes
}

\author{
Mahima Swamy ${ }^{1}$, Gabrielle M Siegers ${ }^{1,2}$, Gina J Fiala ${ }^{1,3}$, Eszter Molnar ${ }^{1}$, Elaine P Dopfer ${ }^{1}$, Paul Fisch, \\ Burkhart Schraven ${ }^{5}$, Wolfgang WA Schamel ${ }^{1 *}$
}

\begin{abstract}
Background: Studying the stoichiometry and intracellular trafficking of the T cell antigen receptor (TCR) is pivotal in understanding its mechanisms of activation. The $\alpha \beta T C R$ includes the antigen-binding TCR $\alpha \beta$ heterodimer as

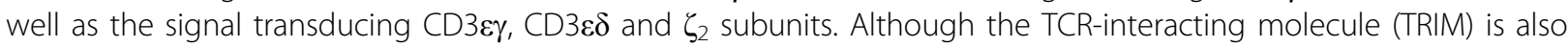
part of the $\alpha \beta T C R$ complex, it has not been included in most reports so far.

Results: We used the native antibody-based mobility shift (NAMOS) assay in a first dimension (1D) blue native (BN)-PAGE and a 2D BN-/BN-PAGE to demonstrate that the stoichiometry of the digitonin-solublized TRIMcontaining $\alpha \beta T C R$ is TCR $\alpha \beta C D 3 \varepsilon_{2} \gamma \delta \zeta_{2} T R I M_{2}$. Smaller $\alpha \beta T C R$ complexes possess a TCR $\alpha \beta C D 3 \varepsilon_{2} \gamma \delta \zeta_{2}$ stoichiometry. Complexes of these sizes were detected in T cell lines as well as in primary human and mouse T cells. Stimulating the $\alpha \beta T C R$ with anti-CD3 antibodies, we demonstrate by confocal laser scanning microscopy that CD3 $\varepsilon$ colocalizes with $\zeta$ and both are degraded upon prolonged stimulation, possibly within the lysosomal compartment. In contrast, a substantial fraction of TRIM does not colocalize with $\zeta$. Furthermore, TRIM neither moves to lysosomes nor is degraded. Immunoprecipitation studies and BN-PAGE indicate that TRIM also associates with the $\gamma \delta T C R$.
\end{abstract}

Conclusions: Small $\alpha \beta T C R$ complexes have a TCR $\alpha \beta C D 3 \varepsilon_{2} \gamma \delta \zeta_{2}$ stoichiometry; whereas those associated with one TRIM dimer are TCR $\alpha \beta C D 3 \varepsilon_{2} \gamma \delta \zeta_{2} T_{R}$ IM $_{2}$. TRIM is differentially processed compared to CD3 and $\zeta$ subunits after $T$ cell activation and is not degraded. The $\gamma \delta$ TCR also associates with TRIM.

\section{Background}

$\mathrm{T}$ cells are classified as $\alpha \beta$ or $\gamma \delta \mathrm{T}$ cells depending on the type of $\mathrm{T}$ cell receptor (TCR) that they express. The $\gamma \delta \mathrm{TCR}$ is expressed on $\gamma \delta \mathrm{T}$ cells and is involved in their development and activation. $\gamma \delta \mathrm{TCR}$ ligands are largely undefined, but include protein and non-protein substances $[1,2]$. The $\alpha \beta$ TCR controls the development and activation of $\alpha \beta \mathrm{T}$ cells, and thus plays a critical role in the adaptive immune system. Its ligands are non-self peptides presented by major histocompatibility complex molecules (MHC) on the surface of antigen presenting cells (APC).

The $\alpha \beta$ TCR consists of TCR $\alpha \beta, \mathrm{CD} 3 \gamma \varepsilon, \mathrm{CD} 3 \delta \varepsilon$ and $\zeta \zeta$ $\left(\mathrm{CD} 247_{2}\right)$ dimers that are non-covalently bound to one another $[3,4]$. The heterodimeric TCR $\alpha \beta$ contains variable immunoglobulin (Ig) domains, which bind to

\footnotetext{
* Correspondence: schamel@immunbio.mpg.de 'Department of Molecular Immunology, Max Planck-Institute of Immunobiology and Institute for Biology III, Albert Ludwigs University Freiburg, Stübeweg 51, 79108 Freiburg, Germany
}

peptide/MHC-complexes. Furthermore, it possesses a transmembrane (TM) region that mediates its association with the CD3 subunits via potentially positively charged amino acids [5-7]. Each CD3 subunit contains an invariable extracellular part, a TM region carrying potentially negatively charged amino acids and a cytoplasmic tail with several signal transduction motifs, including an immunoreceptor tyrosine-based activation motif (ITAM) [8]. The $\zeta$ chain contains only 9 extracellular amino acids buried within the $\alpha \beta$ TCR complex $[9,10]$, a TM region with a potentially negatively charged amino acid that also binds to TCR $\alpha \beta$, and a cytoplasmic tail with three ITAMs. Upon ligand binding to TCR $\alpha \beta$, the CD3 subunits undergo a conformational change [11-14] and the ITAMs within the CD3 cytoplasmic tails and $\zeta$-chains become phosphorylated by protein tyrosine kinases of the Src-family. They subsequently recruit SH2-domain containing intracellular signaling and adapter molecules to the plasma membrane. Hence, while the TCR $\alpha \beta$ heterodimer is responsible for binding 
of the antigen, the CD3 and $\zeta$ subunits of the $\alpha \beta$ TCR serve as signal transducing elements. The $\gamma \delta$ TCR is composed of the same signal transducing elements while carrying the antigen binding $\mathrm{TCR} \gamma \delta$ heterodimer.

In addition to the above molecules, the TCR complex also includes the transmembrane adapter protein TRIM (TCR-Interacting Molecule) [15-17]. The association of TRIM with the $\alpha \beta$ TCR is suggested by the following observations: (i) TRIM co-purifies either with the endogenous $\alpha \beta$ TCR $[16,18]$ or individually expressed $\zeta[18]$ under mild detergent conditions, (ii) TRIM co-caps with CD3 $\varepsilon$ upon antibody-mediated TCR-crosslinking in $\alpha \beta \mathrm{T}$ cells [18] and (iii) overexpression of TRIM leads to enhanced $\alpha \beta$ TCR expression at the cell surface [18]. However, in contrast to CD3 and $\zeta$, TRIM is not mandatory for $\alpha \beta$ TCR assembly and surface expression [19-22].

TRIM is structurally similar to the $\zeta$ chain; it has a short extracellular domain, a long cytoplasmic tail and is a disulfide-linked homodimer. However, its cytoplasmic tyrosine residues are not contained within typical ITAM sequences, but rather within YxxM-motifs that might mediate binding of the p85 subunit of PI3K [16]. TRIM is phosphorylated upon TCR-stimulation by Src protein tyrosine kinases [16] and, together with other transmembrane adapter proteins (e.g. SIT), modulates TCRmediated signals thereby regulating thymocyte development [22,23]. The precise signaling function of TRIM remains elusive, since the TRIM knock-out mouse did not show any phenotype in the assays employed [22].

Using the native antibody-based mobility shift (NAMOS) assay, we recently revealed the stoichiometry of the basic $\alpha \beta$ TCR complex extracted from $\mathrm{T}$ cell membranes which is TCR $\alpha \beta \operatorname{CD} 3 \varepsilon_{2} \gamma \delta \zeta_{2}$ [24-27]. The stoichiometry of the $\gamma \delta \mathrm{TCR}$ is TCR $\gamma \delta \mathrm{CD} 3 \varepsilon_{2} \gamma \delta \zeta_{2}$ (human) and $\mathrm{TCR} \gamma \delta \mathrm{CD} 3 \varepsilon_{2 \gamma 2 \zeta_{2}}$ (mouse) [28]. However, in these studies we did not look for TRIM-containing TCR complexes. Therefore, the present study was aimed at deciphering the stoichiometry of TRIM-containing TCRs. Furthermore, we analyzed the intracellular fate of TRIM upon TCR-stimulation in comparison with that of other components of the TCR complex.

\section{Results}

The $\alpha \beta T C R$ forms two complexes, one of which contains TRIM

The TCR complex of the TCR $\alpha \beta \operatorname{CD} 3 \varepsilon_{2} \gamma \delta \zeta_{2}$ stoichiometry resolves in $\mathrm{BN}-\mathrm{PAGE}$ just below the $\mathrm{f} 1$ marker [24-27]. We noticed that digitonin-purified human TCRs often showed a second larger complex in BNPAGE [25]. To characterize this complex biochemically, using digitonin we purified the $\alpha \beta$ TCR from the 31.13scTCR $\beta$ T cell line [25], which expresses a single chain-tagged TCR $\beta$ subunit (scTCR $\beta$ ) [11]. The sc-tag binds nitrophenol (NP) with moderate and nitroiodophenol (NIP) with high affinity [29]. By binding to NPcoupled columns and subsequently eluting the bound complexes with free NIP, we purified the scTCR in its native form. After separation by BN-PAGE, the eluted $\alpha \beta$ TCR complexes were detected with an anti- $\zeta$ antiserum. Figure 1A, lane 1 shows that under these experimental conditions two distinct $\alpha \beta$ TCRs were detected. Note that the $\alpha \beta$ TCR from Jurkat cells showed the
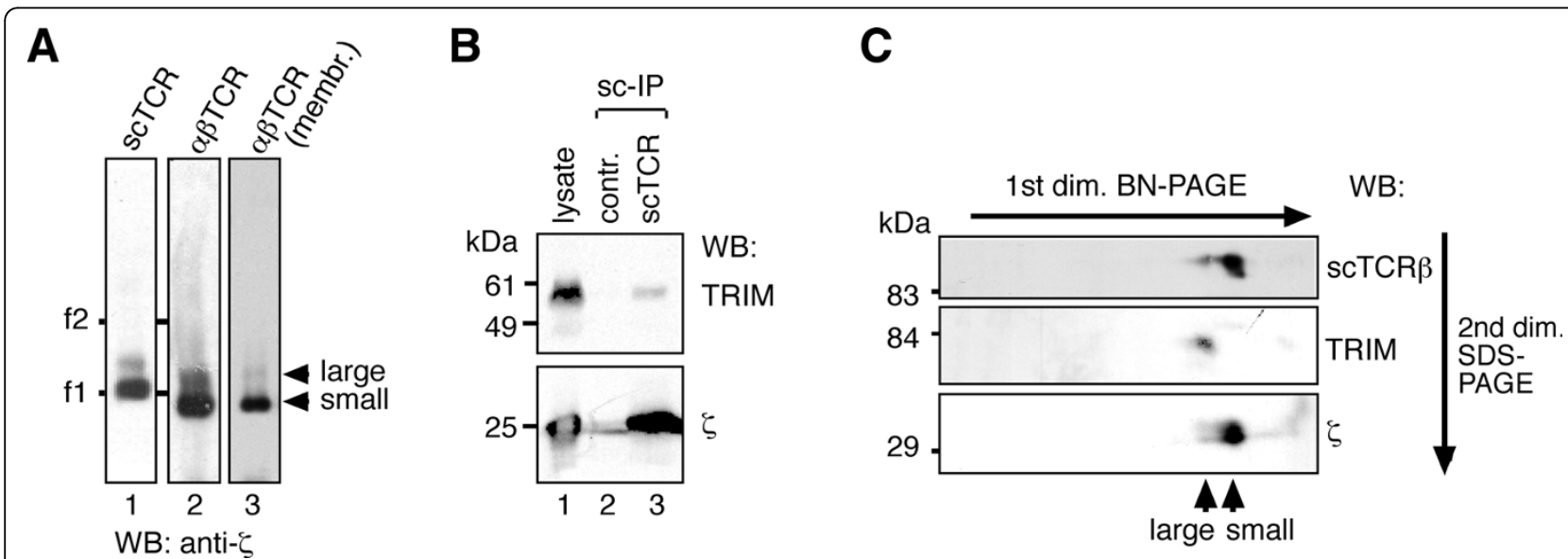

Figure 1 The large $\boldsymbol{\alpha} \beta$ TCR complex contains TRIM. (A) The single chain-tagged TCR (sCTCR) was purified from digitonin-lysed $31.13 s C T C R \beta$ cells using NP-sepharose affinity columns and free NIP for elution (lane 1). Pervanadate-stimulated Jurkat cells were lysed in digitonin and $\alpha \beta$ TCRs purified with anti-phosphotyrosine antibodies followed by phenylphosphate elution (lane 2). Membranes from Jurkat cells were lysed in digitonin (lane 3). TCRs were separated by BN-PAGE and immunoblotted against $\zeta$. The marker protein was ferritin in its $24-$ and 48 -meric forms (f1: 440 kDa, f2: 880 kDa). (B) 31.13scTCR (lanes 1 and 3) and Jurkat (lane 2) cell lysates were subjected to affinity purification on NP-sepharose columns. The purified proteins were separated on a reducing SDS-PAGE; TRIM and $\zeta$ were detected by immunoblotting. (C) NP-purified sCTCRs were separated by $1 \mathrm{D}$ BN-PAGE and 2D non-reducing SDS-PAGE. Immunoblotting was done against sCTCR $\beta$, TRIM and $\zeta$. 
same two bands, although smaller in size due to the absence of the sc-tag (lanes 2 and 3). The larger complex was stimulation-independent, since it was present in pervanadate-stimulated (lane 2) as well as in unstimulated (lane 3) Jurkat cells. The increase in molecular weight for the larger complexes indicated an additional mass of 50 to $100 \mathrm{kDa}$ (not shown).

TRIM forms disulfide-linked dimers of $60 \mathrm{kDa}$ and noncovalently associates with the TCR $[16,18]$. To test whether TRIM is associated with the sc $\alpha \beta$ TCR, we lysed 31.13scTCR $\beta$ or Jurkat cells in digitonin and affinity-purified $\alpha \beta$ TCR complexes with NP-sepharose. The purified material was separated on a reducing SDSPAGE. TRIM and $\zeta$ were detected by Western blotting. The untagged $\alpha \beta$ TCR from Jurkat cells did not bind to NP-sepharose and thus TRIM was not detected (lane 2); however, both TRIM and $\zeta$ were purified from scTCR lysates, (Figure $1 \mathrm{~B}$, lane 3 ), indicating that TRIM associated to the scTCR. This is in line with the binding of TRIM to $\alpha \beta$ TCRs $[16,18]$.

Next we tested whether TRIM was part of the larger $\alpha \beta T C R$ complex seen in BN-PAGE. To this end, the two scTCR complexes in the BN-PAGE were further separated by a second dimension non-reducing SDSPAGE and analysed by anti-TCR $\beta$ and anti- $\zeta$ Western blotting. This approach revealed that both small and large $\alpha \beta$ TCR-complexes contained TCR $\beta$ and $\zeta$ (Figure $1 C$, upper and lower panel). The signal detected by antiTCR $\beta$ was at $90 \mathrm{kDa}$ and that detected by anti- $\zeta$ was at $35 \mathrm{kDa}$, corresponding to the TCR $\alpha \beta$ and $\zeta \zeta$ dimers, respectively. Immunoblotting against TRIM revealed that TRIM dimers of 50-60 $\mathrm{kDa}$ were solely present in the larger $\alpha \beta$ TCR complex. Thus, two $\alpha \beta$ TCR complexes exist in digitonin lysates, one with TRIM and one without.

\section{Stoichiometry of TRIM-containing $\alpha \beta T C R$ complexes}

The NAMOS assay can be used to assess stoichiometries of multiprotein complexes $[25,26]$. When the NAMOS assay was performed using anti-TRIM antibodies, we found that part of the $\alpha \beta$ TCR shifted once at low antibody concentrations (Figure $2 \mathrm{~A}$, band a), indicating one antibody bound to the $\alpha \beta T C R$. With high amounts of anti-TRIM antibody, a second shift was generated (band b) that likely contains two anti-TRIM antibodies. Therefore, two anti-TRIM binding sites and thus two copies of the TRIM protein are present, indicating that one TRIM homodimer [16] is a component of the large $\alpha \beta$ TCR complex.

Next, we modified the NAMOS-assay, using it in a second dimension (2D) BN-PAGE to also assess the amount of CD3 $\varepsilon$ within the complex. The presence of two $\alpha \beta T C R$ complexes precludes the use of the NAMOS-assay in a 1D BN-PAGE, since both large and small $\alpha \beta$ TCR complexes would shift, resulting in several bands, which would be difficult to assign. We separated the two $\alpha \beta$ TCRs by BN-PAGE, and subsequently let antibody bind during a further separation by $2 \mathrm{D} \mathrm{BN}$ PAGE. We tested various experimental conditions to bring the antibody into contact with the complexes inside the first dimension BN-PAGE (not shown). The protocol that worked best was to load varying amounts of antibody onto the 2D gel and allow it to enter the stacking gel by brief electrophoresis. Subsequently, the

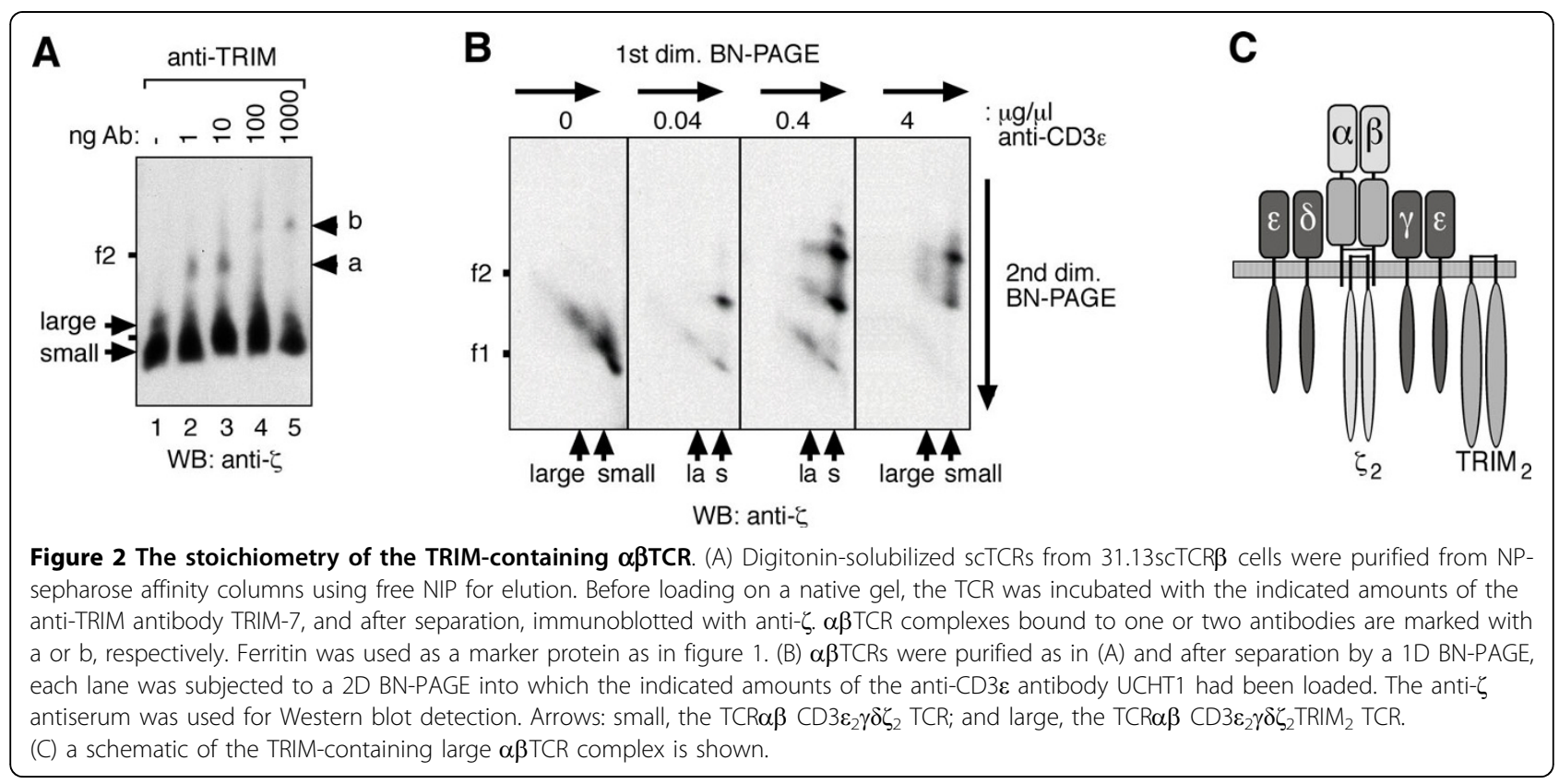


$1 \mathrm{D}$ gel slices containing the $\alpha \beta$ TCR complexes were placed over the 2D stacking gel and electrophoresis was continued. When we applied this 2D BN-/BN-PAGE NAMOS-assay to the $\alpha \beta$ TCRs using an anti-CD3 $\varepsilon$ antibody, we detected two shifts for both complexes (Figure $2 \mathrm{~B})$, indicating that both contained two CD3e subunits. A third shift evident at $0.4 \mu \mathrm{g} / \mu \mathrm{l}$ anti-CD3 $\varepsilon$ is attributed to two $\alpha \beta$ TCR complexes crosslinked by one antibody, a phenomenon occurring at non-saturating antibody concentrations (Figure 2B, 3rd panel). The large $\alpha \beta$ TCR complex thus contains TCR $\alpha \beta$ (Figure $1 \mathrm{C}$ ), $\zeta_{2}$ (Figures 1 and 2 ), one TRIM homodimer (Figure $2 \mathrm{~A}$ ) and two CD $3 \varepsilon$ (Figure $2 B$ ). Since CD $3 \varepsilon$ always pairs with CD $3 \gamma$ and $C D 3 \delta$ [3] it can be concluded that the TRIM-containing $\alpha \beta$ TCR has a stoichiometry of TCR $\alpha \beta$ CD3 $\varepsilon_{2} \gamma \delta \zeta_{2} \mathrm{TRIM}_{2}$ (Figure 2C).

\section{TRIM is loosely associated with the $\alpha \beta T C R$}

We have observed the larger TRIM-containing $\alpha \beta T C R$ in BN-PAGE over many years. One example is shown in figure 3, where we have used the scTCR (Figure 3A). Depending on the experiment, the proportion of TRIMcontaining $\alpha \beta$ TCRs can vary from $50 \%$ of all $\alpha \beta$ TCRs (lane 1) to undetectable amounts (lane 4). The reasons for this inconsistency might include: different lots of the detergent digitonin used for $\alpha \beta T C R$ extraction and

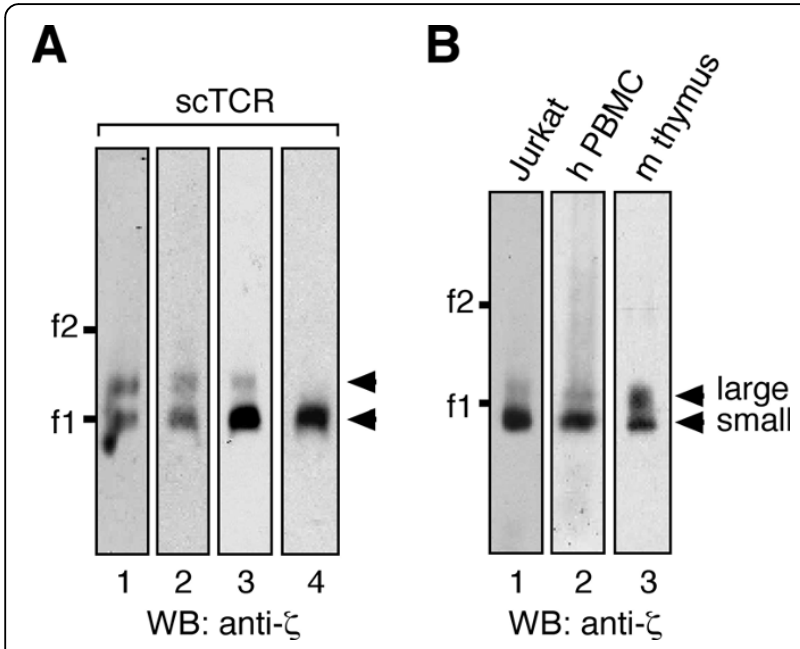

Figure 3 Primary cells express the TRIM-containing $\alpha \beta T C R$. (A) 31.13scTCR $\beta$ cells were lysed with digitonin on different days using different lots of digitonin. scTCRs were purified from NP-sepharose affinity columns using free NIP for elution. TCRs were separated by BN-PAGE and immunoblotted against $\zeta$. (B) TCRs were purified from pervanadate-stimulated Jurkat cells as in figure 1A (lane 1). Membranes were prepared from human PBMC and lysed in digitonin (lane 2). Murine thymocytes were stimulated with pervanadate and and TCRs purified with anti-phosphotyrosine antibodies followed by phenylphosphate elution (lane 3). TCRs were separated by BN-PAGE and immunoblotted against $\zeta$. Ferritin was used as a marker protein as in figure 1. washing, different detergent/lipid ratios and slightly different purification and separation conditions. The large TRIM- $\alpha \beta$ TCR band has a defined size, indicating that it contains a specific protein complex and is not an artefact caused by protein aggregation. The observation that TRIM is easily lost from the $\alpha \beta$ TCR complex shows that TRIM is loosely associated and that a large percentage of $\alpha \beta$ TCRs might contain TRIM in the living cell.

\section{The TRIM-containing $\alpha \beta T C R$ is present in primary T cells}

To extend our studies to primary cells from human and mouse, we used human peripheral blood $\mathrm{T}$ lymphocytes as well as murine thymocytes (Figure 3B, lanes 2 and 3). Jurkat cells served as a control (lane 1). Digitonin-solubilized $\alpha \beta$ TCR complexes were separated by BN-PAGE. The larger $\alpha \beta$ TCR complex, that most likely includes TRIM, was present in primary cells. This is in line with our earlier data showing that TRIM co-caps with CD3 in primary human cells [18] and that TRIM is expressed in murine thymocytes [30].

\section{TRIM is also a component of the $\boldsymbol{\gamma} \delta$ TCR complex}

We are also interested in the $\gamma \delta$ TCR complex. We have shown that the most abundant human $\gamma \delta$ TCR after digitonin extraction has a stoichiometry of $\operatorname{TCR} \gamma \delta$ $\mathrm{CD} 3 \varepsilon_{2} \gamma \delta \zeta_{2}$ [28]. To test whether a second larger complex of the $\gamma \delta$ TCR is detected in BN-PAGE, we analyzed digitonin-purified $\gamma \delta$ TCRs from the human $\gamma \delta$ T cell line Peer or from a human $\gamma \delta \mathrm{T}$ cell clone. Peer cells express a $\gamma \delta \mathrm{TCR}$ containing the TCR $\gamma 2$ protein and the clone a $\mathrm{V} \gamma 9 \mathrm{~V} \delta 2$ receptor (data not shown) including the TCR $\gamma 1$ protein. Separated $\gamma \delta$ TCRs were detected by Western blotting using anti- $\zeta$ antibodies (Figure 4A). Under these experimental conditions two distinct $\gamma \delta$ TCRs were detected. The size difference is likely due to the incorporation of one TRIM dimer in the larger complex. To confirm that TRIM binds the $\gamma \delta$ TCR, we immunopurified the $\gamma \delta$ TCR from lysates of Peer $\gamma \delta \mathrm{T}$ cells using anti-TCR specific antibodies. Complexes were separated by non-reducing SDS-PAGE; TRIM and $\zeta$ were detected by Western blotting (Figure 4B). In control samples, TRIM was absent in anti-TCR $\beta$ precipitates prepared from Peer cells (lane 3) and anti-TCR $\gamma \delta$ precipitates from Jurkat cells (lane 9). However, TRIM was found in anti-CD3 $\varepsilon$, anti-TCR $\gamma \delta$ and anti- $\zeta$ immuno-isolates of Peer cells (lanes 1, 2 and 5) as well as anti-CD3 $\varepsilon$, anti-TCR $\alpha \beta$ and anti- $\zeta$ immuno-isolates of Jurkat T cells (lanes 7, 8 and 11). Thus, TRIM also associates with the $\gamma \delta \mathrm{TCR}$, independent of which TCR $\gamma$ chain is used.

\section{Intracellular fate of TRIM after $\alpha \beta T C R$ stimulation}

Upon binding of peptide/MHC-complexes the $\alpha \beta T C R$ is endocytosed and degraded in lysosomes [31]. To assess 
A

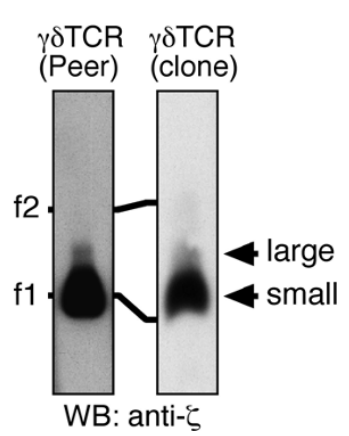

B

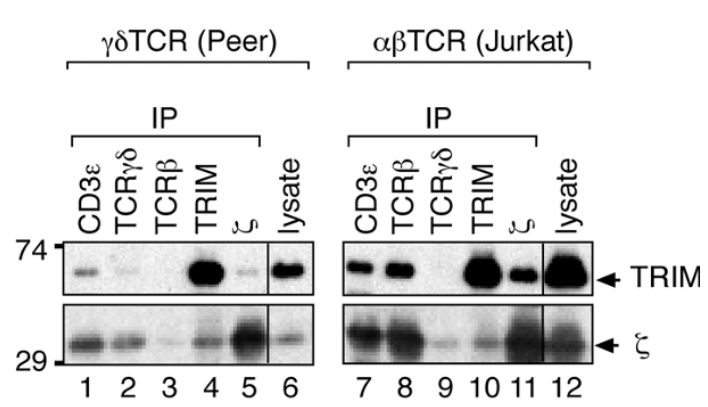

Figure 4 TRIM is part of the $\boldsymbol{\gamma} \boldsymbol{\delta} T C R$ complex. (A) The human $\gamma \delta$ T cell line Peer (left) and a human $V \boldsymbol{\gamma} 9 \mathrm{\delta} 2$ clone (right) were pervanadatestimulated and lysed in digitonin. $\gamma \delta$ TCRs were purified with anti-phosphotyrosine antibodies followed by phenylphosphate elution. TCRs were separated by BN-PAGE and immunoblotted against $\zeta$. Ferritin was used as a marker protein as in figure 1. (B) Peer $\gamma \delta$ and Jurkat $\alpha \beta$ T cells were lysed and immunoprecipitations performed with antibodies recognizing the indicated proteins (lanes $1-5$ and 7 - 11). Purified proteins, as well as lysates (lanes 6 and 12), were separated by non-reducing SDS-PAGE. TRIM and $\zeta$ were detected by immunoblotting.

whether TRIM is also internalized and degraded upon T-cell activation, we stimulated human peripheral blood $\mathrm{T}$ lymphocytes with anti-CD $3 \varepsilon \mathrm{mAb}$ and subsequently investigated the subcellular localization of TRIM by means of confocal laser scanning microscopy. As previously reported, TRIM showed strong capping at the $\mathrm{T}$ cell surface after one hour of $\alpha \beta$ TCR stimulation (Figure $5 \mathrm{~A}$, upper panel). Similarly, $\alpha \beta$ TCR stimulation induced capping of the $\zeta$ chain (lower panel).

To further assess the relationship between $\mathrm{CD} 3 \varepsilon, \zeta$ and TRIM after $\alpha \beta$ TCR stimulation, we studied the colocalization of $\zeta / C D 3 \varepsilon$ and $\zeta / T R I M$ in capped cells by means of confocal laser scanning microscopy. Confirming the tight interaction between the two molecules within the $\alpha \beta$ TCR complex, $\zeta$ and $\mathrm{CD} 3 \varepsilon$ showed an almost $100 \%$ co-localization after 1 hour of $\mathrm{T}$ cell activation (Figure $5 \mathrm{~B}$, left panel). In marked contrast, under the same experimental conditions, we only observed a partial colocalization between TRIM and $\zeta$ (right panel). This finding suggested that the intracellular fate and processing of TRIM differs from that of the other $\alpha \beta$ TCR components. This assumption was further substantiated by the finding that TRIM did not colocalize with the lysosomal marker Lamp1 at early or late time points of $\mathrm{T}$ cell activation (Figure 6A). Furthermore, while the fluorescence signals for both $\mathrm{CD} 3 \varepsilon$ and $\zeta$ were almost completely lost after prolonged stimulation (due to lysosomal degradation), significant amounts of TRIM
A

unstim.
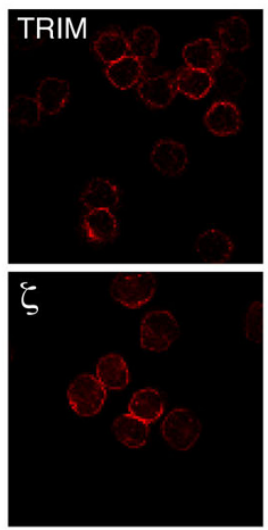

B

$1 \mathrm{~h}$

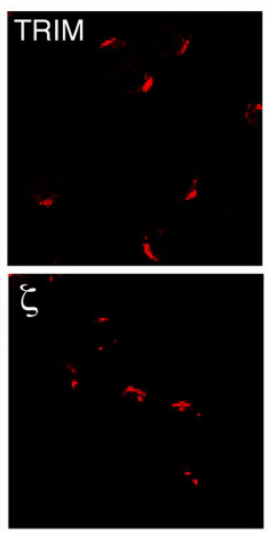

green: $\mathcal{\zeta}$

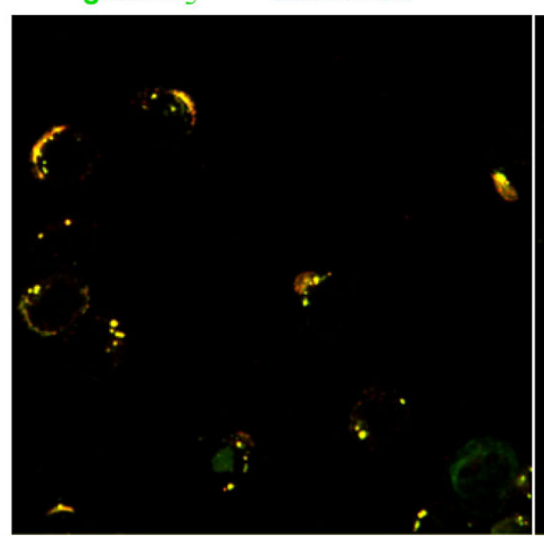

green: $\zeta$

red: TRIM

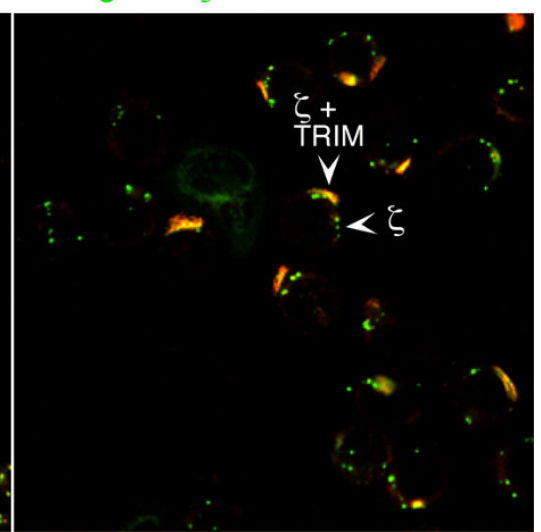

Figure 5 Colocalization of TRIM with CD3 $\varepsilon$ and $\zeta \mathbf{1}$ hour post- $\alpha \beta$ TCR activation. (A) Human peripheral blood T cells were incubated with the $\operatorname{lgM}$ anti-CD3 $\varepsilon$ mAb 2Ad2a2 for 1 hour or left unstimulated. Cells were fixed and the subcellular localization of TRIM and $\zeta$ was determined

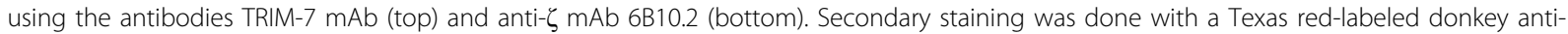
mouse antiserum. (B) Cells were stimulated as in (A), then fixed and the subcellular localization of $\zeta$ detected using FITC-labelled anti- $\zeta$ mAb 6B10.2. CD3 $\varepsilon$ was visualized using Texas Red-labelled goat anti-mouse IgM (left) and a biotinylated TRIM-4 mAb followed by Texas Red-coupled streptavidin were used to detect TRIM (right). 


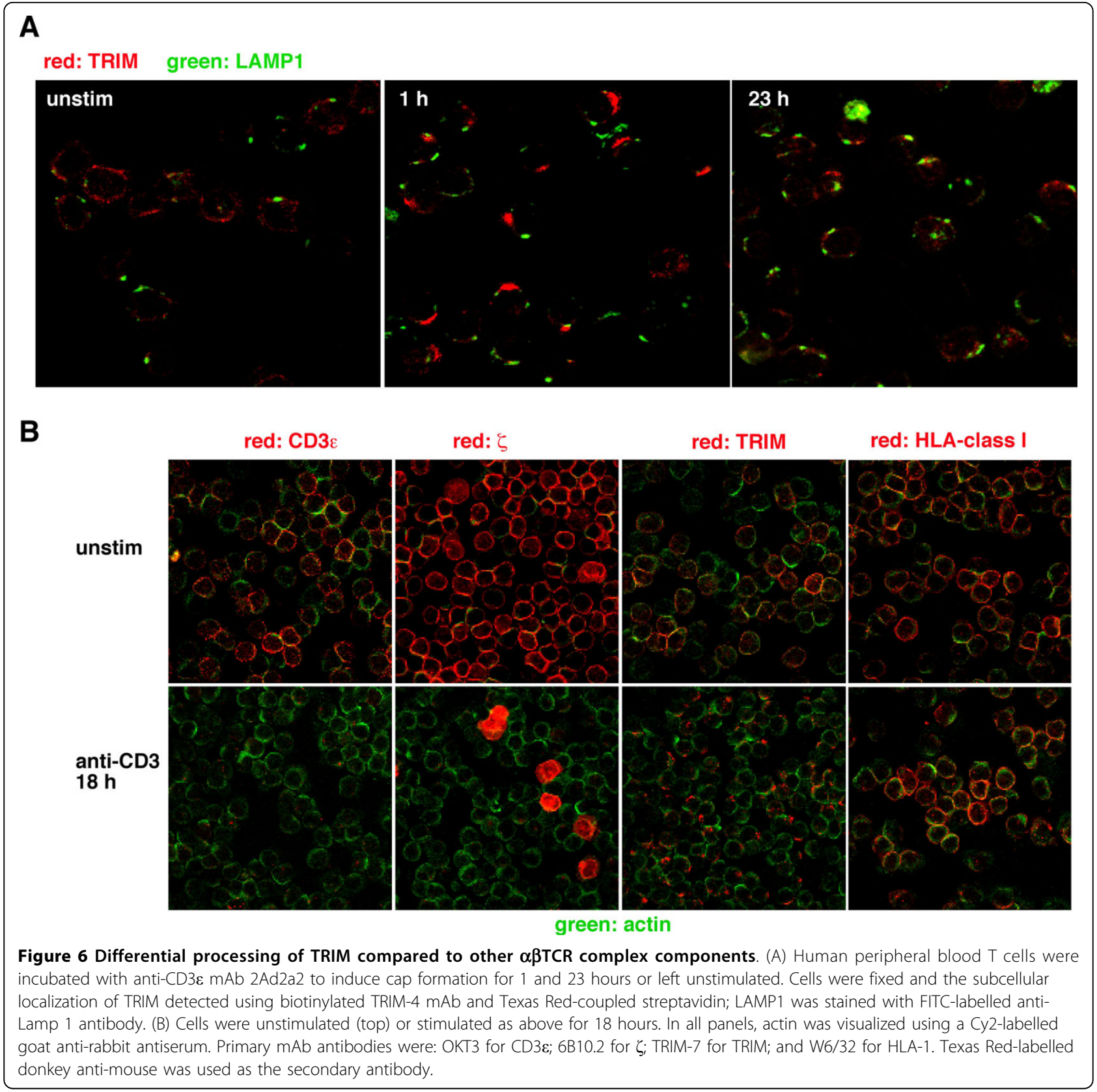

were still detectable even after 18 hours of $\mathrm{T}$ cell activation (Figure 6B). Thus, despite its association with the $\alpha \beta T C R$ complex, upon $\mathrm{T}$ cell activation the intracellular processing of TRIM differs from that of the other $\alpha \beta T C R$ components.

\section{Discussion}

The stoichiometry of the TCR complex has been extensively studied [4]. However, the TRIM protein, known to associate with the TCR, was not included in these studies. Using the NAMOS assay [26], we show that in digitonin two $\alpha \beta T C R$ complexes exist with the stoichiometries TCR $\alpha \beta \quad \mathrm{CD} 3 \varepsilon_{2} \gamma \delta \zeta_{2}$ and $\mathrm{TCR} \alpha \beta$ CD $3 \varepsilon_{2} \gamma \delta \zeta_{2} \mathrm{TRIM}_{2}$. Thus, two TRIM molecules associate with some $\alpha \beta$ TCR complexes. This is in line with the dimeric nature of TRIM [16].

In digitonin detergent lysates, most $\alpha \beta$ TCRs did not include TRIM. On one hand, this could indicate that most $\alpha \beta$ TCRs lack TRIM on the cell surface. This is possible, since TRIM is not required for surface expression of the $\alpha \beta$ TCR [19-22], but still might be included when present. On the other hand, TRIM might be part of all $\alpha \beta \mathrm{TCR}$ complexes in T cells, but partially dissociates under our detergent conditions. The TCR $\alpha \beta$ 
$\mathrm{CD} 3 \varepsilon_{2} \gamma \delta \zeta_{2}$ stoichiometry is held together by potentially charged amino acids in the transmembrane regions of its subunits [7]. TRIM does not have such amino acids in its transmembrane region [16] and thus might associate less stably with the rest of the $\alpha \beta$ TCR. Indeed, our data suggest that TRIM is loosely associated with the $\alpha \beta$ TCR and might be partially (or completely) lost upon purification of the complex.

We have previously shown (and confirmed here) that TRIM co-caps and co-modulates with the $\alpha \beta T C R$. Our early studies demonstrated that the reduction of TRIM expression in TCR-modulated T cells was less severe than the reduction of $\mathrm{CD} 3 \varepsilon$ and $\zeta$ expression. Here, we have extended these observations by showing that the intracellular fate of TRIM differs from that of the other $\alpha \beta$ TCR components. Indeed, upon prolonged mAbmediated stimulation of $\alpha \beta \mathrm{T}$ cells, CD3 $\varepsilon$ and $\zeta$ were no longer detectable by microscopy, most likely due to their degradation within the lysosomal compartment [31-34]. Moreover, confocal laser scanning microscopy revealed an almost complete colocalization of CD3 $\varepsilon$ and $\zeta$ in capped $T$ cells. In marked contrast, a substantial fraction of TRIM did not colocalize with $\zeta$ in these cells. Even after 18 hours of stimulation, a substantial fraction of intracellular TRIM was still detectable within $\mathrm{T}$ cells and thus was not degraded. These findings were further corroborated by the observation that upon $\mathrm{T}$ cell activation TRIM is not targeted to lysosomes (as evidenced by lack of colocalization with the lysosomal marker Lamp1).

The CD3, $\zeta$ and TRIM molecules contain the YxxL/I internalization signal [35] that might target proteins for lysosomal degradation [31]. However, not all YxxL/I containing proteins are degraded. In case of the $\alpha \beta T C R$ it was shown that the Casitas B-lineage lymphoma proteins $\mathrm{c}-\mathrm{Cbl}$ and $\mathrm{Cbl}-\mathrm{b}$ (RING-type E3 ubiquitin ligases) together with $\mathrm{Cbl}$ adaptor proteins Src-like adaptor protein SLAP and $\zeta$-associated protein ZAP70, target internalized $\zeta$ chains to degradation by lysosomes [36-39]. Thus, they are involved in $\alpha \beta$ TCR downmodulation by inhibiting recycling of internalized $\alpha \beta$ TCRs. LAPTM5, a lysosomal protein, is also involved in this degradation pathway, since it binds to ubiquitinylated $\zeta$, but not to CD3 $\varepsilon$, CD3 $\gamma$ or CD3 $\delta$ [34]. Our observation that TRIM does not co-localize with $\zeta$ and is not degraded might be because TRIM is not bound by SLAP, Cbl, ZAP70 or LAPTM5. Indeed. TRIM does not contain any ITAM to which ZAP70 binds.

The double leucine (LL) internalization motif in CD3 $\gamma$ and $\mathrm{CD} 3 \delta$, but not in $\zeta$, CD3 $\varepsilon$ or TRIM, does not target for degradation $[32,40]$. Thus, these signals cannot be the reason for differential targeting of TRIM compared to $\mathrm{CD} 3$ and $\zeta$. However, the transmembrane regions, containing sorting motifs in the case of CD3 $\varepsilon$ and $\zeta$ $[41,42]$, are not conserved between CD3/ $\zeta$ and TRIM. This region of CD3 and $\zeta$ is negatively charged, but neutral in TRIM. Thus, this might be the reason for their differential fates after internalization. In conclusion, while TRIM is a component of large $\alpha \beta$ TCR complexes on the $\mathrm{T}$ cell surface, it is differentially processed after $\mathrm{T}$ cell activation.

TRIM binds to the $\zeta$ subunit of the $\alpha \beta$ TCR [18]. Since the $\gamma \delta$ TCR also contains $\zeta$, we tested whether TRIM might associate with the $\gamma \delta$ TCR and thereby give rise to a second larger detectable $\gamma \delta$ TCR. This was indeed the case as shown by co-immunopurifications and BNPAGE analysis. The small human $\gamma \delta$ TCR complex has a stoichiometry of $\mathrm{TCR} \gamma \delta \mathrm{CD} 3 \varepsilon_{2} \gamma \delta \zeta_{2}$ [2,28]; the larger complex likely has a TCR $\gamma \delta \mathrm{CD} 3 \varepsilon_{2} \gamma \delta \zeta_{2} \mathrm{TRIM}_{2}$ stoichiometry. At the double negative stage of $\mathrm{T}$ cell development, TRIM is already expressed together with the preTCR [30], which also includes $\zeta$. Therefore, it is likely that TRIM also associates with the pre-TCR complex. It remains to be seen whether TRIM processing in the context of pre-TCR and $\gamma \delta$ TCR activation is similar to that observed with the $\alpha \beta$ TCR complex.

Finally, we present an extension of the NAMOS-assay to 2D BN-/BN-PAGE. The multiprotein complexes of interest are first separated by standard BN-PAGE. Then an antibody to a certain subunit $(\mathrm{X})$ is added and a $2 \mathrm{D}$ BN-PAGE is applied. Thus, each individual complex is separately shifted due to the binding of the antibody to $\mathrm{X}$ in the second dimension. From the spot patterns of the $2 \mathrm{D}$ gel, the number of $\mathrm{X}$ molecules per complex can be deduced separately for each complex. Using this method, we show that two CD3 $\varepsilon$ molecules are present in both the small and the large $\alpha \beta$ TCR complex. We tested several protocols and determined how to best add the antibody of interest to the TCR complex present within the 1D BN-PAGE (see Methods). A NAMOSassay 2D BN-/BN-PAGE extends the applicability of the assay to include multiprotein complexes in which a protein exists in more than one complex.

\section{Conclusions}

Here we describe four main results: 1 . A novel method to study stoichiometries in 2D BN-/BN-PAGE, in order to define stoichiometries of multiprotein complexes even if they are present in a mixture; 2 . The $\alpha \beta$ TCR complex is found in two forms: TCR $\alpha \beta \operatorname{CD} 3 \varepsilon_{2} \gamma \delta \zeta_{2-}$ $\mathrm{TRIM}_{2}$ and TCR $\alpha \beta \mathrm{CD} 3 \varepsilon_{2} \gamma \delta \zeta_{2}$, not including TRIM; 3. After stimulation, the $\alpha \beta$ TCR complex is internalized. CD3 subunits are then degraded in lysosomes, however, TRIM is not subject to this trafficking route and remains intact; 4 . TRIM is also associated with the $\gamma \delta \mathrm{TCR}$. 


\section{Methods \\ Cells}

Jurkat, Peer and 31.13scTCR $\beta$ T cells were maintained in RPMI 1640 (GIBCO BRL) medium supplemented with 5\% FCS, as previously described [25]. Peripheral blood $\mathrm{T}$ lymphocytes were prepared by E-rosetting from freshly drawn venous blood of healthy donors as described elsewhere [43]. Mouse thymocytes were prepared as described [44]. The human $\gamma \delta \mathrm{T}$ cell clone was generated from blood as before [28] and was named PF55. PF55 expresses a V $\gamma 9$ V $\delta 2$ TCR (data not shown).

\section{Antibodies}

The following monoclonal and polyclonal antibodies (Abs) were used for immunoprecipitation, confocal laser scan microscopy, Western blotting, and indirect immunofluorescence as indicated: HLA-I (W6/32, for flow cytometry analysis and cap formation); anti- $\zeta$ monoclonal antibody (mAb), clone 6B10.2 (Santa Cruz Biotechnology, Inc., for confocal laser scan analysis and immunoprecipitation); CD3 $\varepsilon$ mAb OKT3 (for co-capping and flow cytometry analysis); CD3 $\varepsilon$ clone $2 \mathrm{Ad} 2 \mathrm{a} 2$ (IgM; donated by Dr. E. Reinherz, Dana Farber Cancer Center, Boston, MA, for modulation experiments). The rabbit anti- $\zeta$ antiserum 448 has been described previously [45]. The anti-TRIM mouse mAb TRIM-7 has also been previously described [18]. Affinity-purified polyclonal anti-TRIM antiserum was prepared as described elsewhere $[16,18]$ and used at $5-10 \mu \mathrm{g} / \mathrm{ml}$ for confocal laser scan analysis.

\section{Cell lysates and protein purification}

To purify the TCR for subsequent BN-PAGE or NAMOS assays, $10^{7}$ Jurkat or Peer T cells were incubated in 200 $\mu \mathrm{M}$ pervanadate $(8.8 \mu \mathrm{l} 23 \mathrm{mM}$ sodium orthovanadate plus $1.2 \mu \mathrm{l} 30 \% \mathrm{H}_{2} \mathrm{O}_{2}$ ) at $37^{\circ} \mathrm{C}$ for $5 \mathrm{~min}$ in $1 \mathrm{ml} \mathrm{RPMI}$ medium to induce tyrosine phosphorylation of the TCR. Cells were lysed in $1 \mathrm{ml}$ lysis buffer $(20 \mathrm{mM}$ TrisHCl pH $8,137 \mathrm{mM} \mathrm{NaCl}, 2 \mathrm{mM}$ EDTA, $10 \%$ glycerol, $10 \mu \mathrm{g} / \mathrm{ml}$ leupeptin, $10 \mu \mathrm{g} / \mathrm{ml}$ aprotinin, $1 \mathrm{mM}$ PMSF, $500 \mu \mathrm{M}$ $\mathrm{Na}_{3} \mathrm{VO}_{4}, 1 \mathrm{mM} \mathrm{NaF}$ and $1 \%$ digitonin). Phosphorylated proteins were purified by incubation with $1 \mu \mathrm{g}$ of the anti-phosphotyrosine Ab 4G10 (Upstate Biotechnology) and $3 \mu$ protein G-Sepharose (Pharmacia) for minimum 6 hours. The beads were washed twice in lysis buffer, once in BN buffer (0.5 M 6-aminohexanoic acid, 12.5 $\mathrm{mM} \mathrm{NaCl}, 10 \%$ glycerol, $2 \mathrm{mM}$ EDTA, $0.5 \%$ digitonin, 20 $\mathrm{mM}$ BisTris pH7.0, $1 \mathrm{mM}$ PMSF) and eluted in $100 \mu \mathrm{l}$ $\mathrm{BN}$ elution buffer (BN buffer including $50 \mathrm{mM}$ phenylphosphate and 1 unit alkaline phosphatase to dephosphorylate the receptor) for a minimum of $30 \mathrm{~min}$ on ice. The scTCR was purified from cell lysates using NP (3-nitro-4-hydroxy-phenylacetate)-conjugated Sepharose, washed three times with BN buffer, and the scTCR eluted in BN-buffer containing $0.5 \mathrm{mM}$ free NIP (NIP-caproic acid, Biosearch Technologies). The eluate was then directly loaded onto a $\mathrm{BN}$ gel, or first incubated with Abs against specific subunits, as detailed below.

\section{Membranes were prepared as described [12]}

For immunoprecipitation of $\alpha \beta$ or $\gamma \delta$ TCRs in figure 4, Jurkat or Peer cells were lysed in $1 \mathrm{ml}$ lysis buffer (as above, but containing $0.5 \%$ Brij-96 instead of digitonin) and purified by incubation with antibodies against CD3 $\varepsilon$ (UCHT1, kind gift of Dr. P Beverley, The Edward Jenner Institute for Vaccine Research, Compton, UK), $\gamma \delta \mathrm{TCR}$ (5A6. E9, Pierce Chemical Co.), TCR $\beta$ (BV8, Pharmingen), TRIM or $\zeta(448$, described above) coupled to protein G-Sepharose for 4 hours. Beads were washed 4 times with lysis buffer, resuspended in non-reducing sample buffer, boiled 5 min and loaded onto SDS-PAGE.

\section{BN-PAGE, SDS-PAGE and immunoblotting}

BN-PAGE gradient gels (4\%-9\%) were prepared using a gradient mixer and the Protean II system from BioRad; the stacking gel was $3.2 \%$ polyacrylamide. Gel composition, pouring procedure and running conditions have been described [46-49]. In short, the loaded gels were run at $4^{\circ} \mathrm{C}$ at $20 \mathrm{~V}$ overnight. For running BN-PAGE, sample buffer was omitted, but samples in $\mathrm{BN}$ elution buffer were carefully overlaid with blue cathode buffer instead (15 mM BisTris pH7.0, $50 \mathrm{mM}$ Tricine and $0.02 \%$ Coomassie blue G250). The marker protein was ferritin in its 24-, 48- and 72 meric forms (f1: $440 \mathrm{kDa}$, f2: $880 \mathrm{kDa}, \mathrm{f} 3: 1320 \mathrm{kDa}$, respectively). Western blotting was done using PVDF membranes and a semi-dry transfer system, at $18 \mathrm{~V}$ for 15 to 20 minutes, with transfer buffer containing 0.1\% SDS. SDS-PAGE was performed using standard protocols. The membranes were developed using the Abs mentioned above and secondary Abs were anti-rabbit IgG coupled to HRPO or goat anti-mouse IgG HRPO from Southern Biotechnology. Visualization was performed using the ECL system.

The NAMOS assay was done as described [26,27]. In brief, the indicated amounts of Abs were added to the samples (10 to $20 \mu \mathrm{l})$, mixed and incubated for 5-30 min on ice before loading them on BN-PAGE. MPCantibody conjugates migrate slower in native gels, such that the shift directly reflects the number of $\mathrm{Ab}$ molecules bound to the complex.

\section{NAMOS (Native antibody-based mobility shift) assay in the second dimension}

Protein complexes are initially separated by a first dimension (1D) BN-PAGE. They are then brought into contact with anti-subunit Abs while inside the gel. Finally, the 
protein complex-antibody supercomplexes are separated by a second dimension (2D) BN-PAGE. We tested several experimental procedures for the formation of supercomplexes. The most successful procedure was to load given amounts of $\mathrm{Ab}$ in a small volume (e.g. $10-20 \mu \mathrm{l})$ into the large pocket of the 2D stacking gel. Using the BioRad minigel equipment, electrophoresis is carried out for a few minutes at $100 \mathrm{~V}$, in order to let the Ab enter $1-2 \mathrm{~mm}$ into the stacking gel. Then the gel run is stopped and the 1D gel lane placed into the large pocket, after which the gel run is continued. During the stacking process, the $\mathrm{Ab}$ comes into contact with the protein complexes and forms supercomplexes that are then separated by the 2D separating gel.

\section{Co-capping Experiments}

Resting peripheral blood $\mathrm{T}$ cells were washed twice with RPMI (without FCS) and adjusted to $7 \times 10^{6}$ cells $/ \mathrm{ml}$ in RPMI 1640. $10 \mu \mathrm{l}$ of this suspension was pipetted onto each reaction field of an adhesion slide (Bio-Rad Laboratories) and allowed to adhere for $10 \mathrm{~min}$ at room temperature. Adherent cells were washed twice with RPMI and the slide was blocked for 15 min with complete culture medium. Cap formation was subsequently induced by incubating the cells for $15 \mathrm{~min}$ with $15 \mu \mathrm{lmAb}$ solution $(10 \mu \mathrm{g} / \mathrm{ml}$ in complete culture medium), followed by two washes with RPMI $1640(50 \mu \mathrm{l}$ per field) and cross-linking of the primary Abs with $15 \mu \mathrm{l}$ Texas redlabeled donkey anti-mouse antiserum (Dianova; diluted 1:50 $\mathrm{vol} / \mathrm{vol}$ in culture medium) for $35 \mathrm{~min}$ at room temperature in the dark. Capped cells were washed twice with $50 \mu \mathrm{l}$ PBS supplemented with $0.2 \% \mathrm{NaN}_{3}$ and then fixed for $3 \mathrm{~min}$ with cold methanol (stored at $-20^{\circ}$ C). The methanol was removed and the cells were fixed for an additional $5 \mathrm{~s}$ with ice-cold acetone. Fixed cells were washed twice with PBS and blocked again for 10 min with $\mathrm{PBS} / 1 \% \mathrm{BSA}$ at room temperature. Subsequently, affinity-purified polyclonal Abs directed at TRIM and CD3 $\varepsilon$ were added $(15 \mu \mathrm{l}$ per field, concentration $10 \mu \mathrm{g} / \mathrm{ml}$ ) and incubated for $30 \mathrm{~min}$ at room temperature in the dark. The Ab-labeled cells were then washed once with PBS/BSA ( $40 \mu \mathrm{l}$ per field) and three times with PBS. $15 \mu \mathrm{l}$ secondary Cy2-labeled donkey anti-rabbit Ab (Dianova) were then added (1:125 vol/vol in $\mathrm{PBS} / 1 \% \mathrm{BSA}$ ) and incubated for $30 \mathrm{~min}$ at room temperature in the dark. Cells were washed once with PBS/ BSA, three times with PBS, and finally at least $5 \mathrm{~min}$ with distilled water. The slides were then covered with a cover slip, which was fixed with Elvanol (Calbiochem) for at least $16 \mathrm{~h}$.

Slides were viewed on a Laser scan microscope (Ernst Leitz $\mathrm{GmbH}$ ) using $570 \mathrm{~nm}$ (red emission) and $508 \mathrm{~nm}$ (green emission) filters, respectively. In general, the magnification was $40 \times$.

\section{Acknowledgements}

We thank Balbino Alarcon, Vaclav Horjesi and Michael Reth for scientific support. This work was funded by the Deutsche Forschungsgemeinschaft through the Emmy Noether program (to W.S.), the SFB620 (to W.S. and P.F.) and FOR521 (to B.S.) and the EU grant FP7/2007-2013 (SYBILLA, to W.S. and B.S.). This study was supported in part by the Excellence Initiative of the German Federal and State Governments (GSC-4, Spemann Graduate School).

\section{Author details}

${ }^{1}$ Department of Molecular Immunology, Max Planck-Institute of Immunobiology and Institute for Biology III, Albert Ludwigs University Freiburg, Stübeweg 51, 79108 Freiburg, Germany. ${ }^{2}$ Cell Therapy Program, Princess Margaret Hospital/Ontario Cancer Institute, 610 University Ave., Toronto, Ontario, M5G 2M9, Canada. ${ }^{3}$ Spemann Graduate School of Biology and Medicine (SGBM), Albert Ludwigs University Freiburg, Albertstraße 19A, 79104 Freiburg, Germany. ${ }^{4}$ Department of Pathology, University of Freiburg Medical Center, 79110 Freiburg, Germany. ${ }^{5}$ Institute of Molecular and Clinical Immunology, Otto-von-Guericke-Universität Magdeburg, Leipziger Strasse 44, 39120 Magdeburg, Germany.

\section{Authors' contributions}

MS carried out most of the biochemical characterization of the TRIMcontaining $\alpha \beta T C R s$ and drafted the manuscript. GMS performed immunoprecipitation studies and helped to draft the manuscript. GJF did BN-PAGE with the $\gamma \delta T C R$. EM and EPD performed biochemical experiments on the $\alpha \beta T C R$. PF generated and cultivated the $\gamma \delta$ T cell clone. BS performed all microscopy experiments and helped to draft the manuscript. WWAS conceived of the study, and participated in its design and coordination and helped to draft the manuscript. All authors read and approved the final manuscript.

\section{Competing interests}

The authors declare that they have no competing interests.

Received: 31 December 2009 Accepted: 18 March 2010

Published: 18 March 2010

\section{References}

1. Hayday AC: $\gamma \delta$ cells: a right time and a right place for a conserved third way of protection. Annu Rev Immunol 2000, 18:975-1026.

2. Siegers GM, Fernandez-Malave E, Fisch P, Regueiro JR, Schamel WW: From structure to disease: differential CD3 usage in the human and mouse $\mathrm{gd}$ $T$ cell antigen receptor determines the impact of CD3 deficiencies on $T$ cell development. Int J Med Biol Frontiers 2008, 14:115-132.

3. Alarcon B, Gil D, Delgado P, Schamel WWA: Initiation of TCR signaling: regulation within CD3 dimers. Immunol Rev 2003, 191:38-46.

4. Alarcon B, Swamy M, van Santen HM, Schamel WW: T-cell antigenreceptor stoichiometry: pre-clustering for sensitivity. EMBO Rep 2006, 7:490-495.

5. Blumberg RS, Alarcon B, Sancho J, McDermott FV, Lopez P, Breitmeyer J, Terhorst C: Assembly and function of the $T$ cell antigen receptor. Requirement of either the lysine or arginine residues in the transmembrane region of the alpha chain. J Biol Chem 1990, 265:14036-14043.

6. Cosson P, Lankford SP, Bonifacino JS, Klausner RD: Membrane protein association by potential intramembrane charge pairs. Nature 1991, 351:414-416.

7. Call ME, Pyrdol J, Wiedmann M, Wucherpfennig KW: The organizing principle in the formation of the T cell receptor-CD3 complex. Cell 2002, 111:967-979.

8. Reth M: Antigen receptor tail clue. Nature 1989, 338:383.

9. Minguet S, Swamy M, Dopfer EP, Dengler E, Alarcon B, Schamel WWA: The extracellular part of zeta is buried in the $T$ cell antigen receptor complex. Immunol Lett 2008, 116:203-210.

10. Minguet $S$, Swamy M, Schamel WWA: The short length of the extracellular domain of zeta is crucial for T cell antigen receptor function. Immunol Lett 2008, 116:195-202

11. Gil D, Schamel WW, Montoya M, Sanchez-Madrid F, Alarcon B: Recruitment of Nck by CD3 epsilon reveals a ligand-induced conformational change essential for T cell receptor signaling and synapse formation. Cell 2002, 109:901-912. 
12. Minguet S, Swamy M, Alarcon B, Luescher IF, Schamel WW: Full activation of the $T$ cell receptor requires both clustering and conformational changes at CD3. Immunity 2007, 26:43-54.

13. Risueno RM, Schamel WW, Alarcon B: T cell receptor engagement triggers its CD3epsilon and CD3zeta subunits to adopt a compact, locked conformation. PLOS ONE 2008, 3:e1747.

14. Minguet S, Schamel WWA: A permissive geometry model for TCR-CD3 activation. Trends Biochem Sci 2008, 33:51-57.

15. Schraven B, Ratnofsky $\mathrm{S}$, Gaumont $\mathrm{Y}$, Lindegger $\mathrm{H}$, Kirchgessner $\mathrm{H}$, Bruyns $\mathrm{E}$, Moebius U, Meuer SC: Identification of a novel dimeric phosphoprotein (PP29/30) associated with signaling receptors in human T lymphocytes and natural killer cells. J Exp Med 1994, 180:897-906.

16. Bruyns E, Marie-Cardine A, Kirchgessner H, Sagolla K, Shevchenko A, Mann M, Autschbach F, Bensussan A, Meuer S, Schraven B: T cell receptor (TCR) interacting molecule (TRIM), a novel disulfide-linked dimer associated with the TCR-CD3-zeta complex, recruits intracellular signaling proteins to the plasma membrane. J Exp Med 1998, 188:561-575.

17. Horejsi V, Zhang W, Schraven B: Transmembrane adaptor proteins: organizers of immunoreceptor signalling. Nat Rev Immunol 2004, 4:603-616

18. Kirchgessner H, Dietrich J, Scherer J, Isomaki P, Korinek V, Hilgert I, Bruyns E, Leo A, Cope AP, Schraven B: The transmembrane adaptor protein TRIM regulates $\mathrm{T}$ cell receptor (TCR) expression and TCR-mediated signaling via an association with the TCR zeta chain. J Exp Med 2001, 193:1269-1284.

19. Berkhout B, Alarcon B, Terhorst C: Transfection of genes encoding the $T$ cell receptor-associated CD3 complex into COS cells results in assembly of the macromolecular structure. J Biol Chem 1988, 263:8528-8536.

20. Hall C, Berkhout B, Alarcon B, Sancho J, Wileman T, Terhorst C: Requirements for cell surface expression of the human TCR/CD3 complex in non-T cells. Int Immunol 1991, 3:359-368.

21. Carson GR, Kuestner RE, Ahmed A, Pettey CL, Concino MF: Six chains of the human T cell antigen receptor. CD3 complex are necessary and sufficient for processing the receptor heterodimer to the cell surface. J Biol Chem 1991, 266:7883-7887.

22. Koelsch U, Arndt B, Reinhold D, Lindquist JA, Juling N, Kliche S, Pfeffer K, Bruyns E, Schraven B, Simeoni L: Normal T-cell development and immune functions in TRIM-deficient mice. Mol Cell Biol 2006, 26:3639-3648.

23. Koelsch U, Schraven B, Simeoni L: SIT and TRIM determine T cell fate in the thymus. J Immunol 2008, 181:5930-5939.

24. Zapata DA, Schamel WW, Torres PS, Alarcon B, Rossi NE, Navarro MN, Toribio ML, Regueiro J: Biochemical differences in the alphabeta $\mathrm{T}$ cell receptor. CD3 surface complex between CD8+ and CD4+ human mature T lymphocytes. J Biol Chem 2004, 279:24485-24492.

25. Schamel WW, Arechaga I, Risueno RM, van Santen HM, Cabezas P, Risco C, Valpuesta JM, Alarcon B: Coexistence of multivalent and monovalent TCRs explains high sensitivity and wide range of response. J Exp Med 2005, 202:493-503.

26. Swamy M, Minguet $S$, Siegers GM, Alarcon B, Schamel WW: A native antibody-based mobility-shift technique (NAMOS-assay) to determine the stoichiometry of multiprotein complexes. J Immunol Methods 2007, 324:74-83

27. Swamy M, Dopfer EP, Molnar E, Alarcon B, Schamel WW: The 450 kDa TCR Complex has a Stoichiometry of alphabetagammaepsilondeltaepsilonzetazeta. Scand J Immunol 2008, 67:418-420, author reply 421.

28. Siegers GM, Swamy M, Fernandez-Malave E, Minguet S, Rathmann $S$, Guardo AC, Perez-Flores V, Regueiro JR, Alarcon B, Fisch P, Schamel WW: Different composition of the human and the mouse gammadelta $T$ cell receptor explains different phenotypes of CD3gamma- and CD3deltaimmunodeficiencies. J Exp Med 2007, 204:2537-2544.

29. Reth M, Imanishi-Kari T, Rajewsky K: Analysis of the repertoire of anti-(4hydroxy-3-nitrophenyl)acetyl (NP) antibodies in C $57 \mathrm{BL} / 6$ mice by cell fusion. II. Characterization of idiotopes by monoclonal anti-idiotope antibodies. Eur J Immunol 1979, 12:1004-1013.

30. Huynh T, Wurch A, Bruyns E, Korinek V, Schraven B, Eichmann K: Developmentally regulated expression of the transmembrane adaptor protein trim in fetal and adult T cells. Scand J Immunol 2001, 54:146-154

31. Alcover A, Alarcon B: Internalization and intracellular fate of TCR-CD3 complexes. Crit Rev Immunol 2000, 20:325-346.
32. Niedergang F, San Jose E, Rubin B, Alarcon B, Dautry-Varsat A, Alcover A Differential cytosolic tail dependence and intracellular fate of T-cell receptors internalized upon activation with superantigen or phorbol ester. Res Immunol 1997, 148:231-245.

33. Valitutti S, Muller S, Salio M, Lanzavecchia A: Degradation of T cell receptor (TCR)-CD3-zeta complexes after antigenic stimulation. J Exp Med 1997, 185:1859-1864.

34. Ouchida R, Yamasaki S, Hikida M, Masuda K, Kawamura K, Wada A, Mochizuki S, Tagawa M, Sakamoto A, Hatano M, Tokuhisa T, Koseki H, Saito T, Kurosaki T, Wang JY: A lysosomal protein negatively regulates surface $T$ cell antigen receptor expression by promoting CD3zeta-chain degradation. Immunity 2008, 29:33-43.

35. Mellman I: Endocytosis and molecular sorting. Annu Rev Cell Dev Biol 1996, 12:575-625.

36. Wang HY, Altman Y, Fang D, Elly C, Dai Y, Shao Y, Liu YC: Cbl promotes ubiquitination of the $\mathrm{T}$ cell receptor zeta through an adaptor function of Zap-70. J Biol Chem 2001, 276:26004-26011.

37. Naramura M, Jang IK, Kole H, Huang F, Haines $D$, Gu H: c-Cbl and Cbl-b regulate $T$ cell responsiveness by promoting ligand-induced TCR downmodulation. Nat Immunol 2002, 3:1192-1199.

38. Myers MD, Dragone LL, Weiss A: Src-like adaptor protein down-regulates $T$ cell receptor (TCR)-CD3 expression by targeting TCRzeta for degradation. J Cell Biol 2005, 170:285-294.

39. Myers MD, Sosinowski T, Dragone LL, White C, Band H, Gu H, Weiss A: Srclike adaptor protein regulates TCR expression on thymocytes by linking the ubiquitin ligase c-Cbl to the TCR complex. Nat Immunol 2006, 7:57-66.

40. Dietrich J, Backstrom T, Lauritsen JP, Kastrup J, Christensen MD, von Bulow F, Palmer E, Geisler C: The phosphorylation state of CD3gamma influences T cell responsiveness and controls T cell receptor cycling. J Biol Chem 1998, 273:24232-24238

41. San Jose E, Borroto A, Niedergang F, Alcover A, Alarcon B: Triggering the TCR complex causes the downregulation of nonengaged receptors by a signal transduction-dependent mechanism. Immunity 2000, 12:161-170.

42. Borroto A, Lama J, Niedergang F, Dautry-Varsat A, Alarcon B, Alcover A: The CD3 epsilon subunit of the TCR contains endocytosis signals. J Immunol 1999, 163:25-31.

43. Schraven B, Roux M, Hutmacher B, Meuer SC: Triggering of the alternative pathway of human $T$ cell activation involves members of the T 200 family of glycoproteins. Eur J Immunol 1989, 19:397-403.

44. Fernandez-Malave E, Wang N, Pulgar M, Schamel WW, Alarcon B, Terhorst C: Overlapping functions of human CD3\{delta\} and mouse CD3 \{gamma\} in \{alpha\}beta T-cell development revealed in a humanized CD3\{gamma\}-deficient mouse. Blood 2006, 108:3420-3427.

45. San Jose E, Sahuquillo AG, Bragado R, Alarcon B: Assembly of the TCR/CD3 complex: CD3 epsilon/delta and CD3 epsilon/gamma dimers associate indistinctly with both TCR alpha and TCR beta chains. Evidence for a double TCR heterodimer model. Eur J Immunol 1998, 28:12-21.

46. Schägger $\mathrm{H}$, von Jagow $\mathrm{G}$ : Blue native electrophoresis for isolation of membrane protein complexes in enzymatically active form. Anal Biochem 1991, 199:223-231.

47. Schamel WW, Reth M: Monomeric and oligomeric complexes of the B cell antigen receptor. Immunity 2000, 13:5-14.

48. Swamy M, Siegers GM, Minguet S, Wollscheid B, Schamel WW: Blue native polyacrylamide gel electrophoresis (BN-PAGE) for the identification and analysis of multiprotein complexes. SCi STKE 2006, 2006:14.

49. Schamel WW: Two-dimensional blue native polyacrylamide gel electrophoresis. Curr Protoc Cell Biol 2008, Chapter 6(Unit 6):10.

doi:10.1186/1478-811X-8-5

Cite this article as: Swamy et al.: Stoichiometry and intracellular fate of TRIM-containing TCR complexes. Cell Communication and Signaling 2010 8:5. 